\title{
La violencia obstétrica en la literatura de las ciencias sociales en América Latina*
}

\section{Obstetric Violence in the Social Science Literature in Latin America}

\section{A violência obstétrica na literatura das ciências sociais na América Latina}

Fecha de recepción: 9 de abril de 2018. Fecha de aprobación: 4 de junio de 2018.

Fecha de publicación: 27 de mayo de 2019

DOI: https://doi.org/10.11144/Javeriana.rgps18-36.volc

\author{
María Mercedes Lafaurie Villamila \\ Universidad El Bosque, Colombia \\ ORCID: 0000-0002-5993-740X \\ Diana Carolina Rubio León \\ Universidad El Bosque, Colombia \\ ORCID: 0000-0002-7672-9776 \\ Alejandro Perdomo Rubio \\ Universidad El Bosque, Colombia \\ ORCID: 0000-0003-1157-4045 \\ Andrés Felipe Cañón Crespo \\ Universidad El Bosque, Colombia \\ ORCID: 0000-0003-4233-3578
}

Cómo citar este artículo: Lafaurie Villamil MM, Rubio León DC, Perdomo Rubio A, Cañón Crespo AF. La violencia obstétrica en la literatura de las ciencias sociales en América Latina. Revista Gerencia y Políticas de Salud. 2019;18(36). https://doi.org/10.11144/Javeriana.rgps18-36.volc

Artículo de investigación

Autor de correspondencia. Correo electrónico: lafauriemaria@unbosque.edu.co 


\section{Resumen}

A partir del análisis de dieciocho estudios realizados por científicos sociales de América Latina, el presente trabajo busca poner en el escenario la "violencia obstétrica" y suscitar reflexión en torno a su conceptualización y sus orígenes, además de retomar las recomendaciones propuestas por las y los autores para un cambio de situación y de proponer nuevas rutas de indagación. Se requiere un cambio de paradigmas en los sistemas de salud, en los procedimientos y en la formación de los profesionales con miras a la humanización de la atención en el campo de la salud de las mujeres y la erradicación de todas las formas de la violencia obstétrica para que sea posible hablar de ejercicio de ciudadanía en ese ámbito; en la investigación social es necesario incluir la perspectiva del personal de salud y profundizar en realidades como la vulnerabilidad social de las mujeres y el aborto.

Palabras clave: violencia contra la mujer, embarazo, parto, personal de salud, salud sexual y reproductiva

\section{Abstract}

Based on an analysis including eighteen studies conducted by social scientists in Latin America, this work intends to bring into the spotlight the "obstetric violence" and encourage the reflection on how to conceptualize it and its background. In addition, this work resumes the recommendations proposed by female and male authors to make a change in the current situation and set out new routes for inquiring on the issue. A paradigm change in the health systems, health procedures, and the education of health professionals intended to the humanization of the health care provided to women as well as the elimination of any kind of obstetric violence is required to make possible that women exercise their rights as citizens in the field of health. The social research should consider the health professional's perspective and deepen in the study of realities like the social vulnerability of women and the abortion.

Keywords: violence against women, pregnancy, labor, health workers, sexual and reproductive health

\section{Resumo}

A partir da análise de dezoito estudos realizados por cientistas sociais da América Latina, o presente trabalho visa colocar no palco a "violência obstétrica" e suscitar a reflexão sobre sua conceituação e origens, além de retomar as recomendações propostas pelas autoras e autores para uma mudança de situação e propor novas rotas de investigação. Uma mudança de paradigmas nos sistemas de saúde, nos procedimentos e na formação dos profissionais com vista à humanização do atendimento no campo da saúde das mulheres e a erradicação de toda forma de violência obstétrica são necessários para que seja possível falar de exercício de cidadania nessa área; na pesquisa social é preciso incluir a perspectiva do pessoal de saúde e aprofundar-se em realidades como a vulnerabilidade social das mulheres e o aborto.

Palavras chave: violência contra a mulher, gravidez, parto, pessoal de saúde, saúde sexual e reprodutiva

\section{Introducción}


El cuerpo de las mujeres posee una gran carga simbólica que ha permeado desde tiempos inmemoriales la atención en salud sexual y reproductiva; son diversas las problemáticas en la salud de las mujeres asociadas con la invisibilización de los sesgos y estereotipos de género que persisten en el sistema biomédico. Más de medio siglo después de haberse iniciado el movimiento por los derechos sexuales y reproductivos, el mundo vuelve los ojos hacia la violencia obstétrica como violencia de carácter institucional estrechamente asociada a la subvaloración del sexo femenino. Su conceptualización y análisis cobra un interés cada vez mayor para las ciencias sociales, en especial en el contexto de América Latina donde, desde mediados de la década del 2000, ha venido avanzando cada vez con mayor fuerza el movimiento que le ha dado vida a su reconocimiento como problema de salud pública. Si bien desde años atrás disciplinas como la antropología médica y la sociología de la salud han venido haciendo lecturas críticas sobre tópicos como la salud de las mujeres, la atención en salud reproductiva, la autonomía del cuerpo y los derechos sexuales y reproductivos, es de manera reciente que la categoría "violencia obstétrica" se incluye en la literatura científica de las ciencias sociales. La violencia obstétrica fue tipificada por primera vez en el ámbito institucional a través de la Ley orgánica sobre el derecho de las mujeres a una vida sin violencia, sancionada en Venezuela y publicada por el Fondo de Población de las Naciones Unidas (UNFPA) en el año 2006 (1), en que se le definió como sigue:

Apropiación del cuerpo y procesos reproductivos de las mujeres por personal de salud, que se expresa en un trato deshumanizador, en un abuso de medicalización y patologización de los procesos naturales, trayendo consigo pérdida de autonomía y capacidad de decidir libremente sobre sus cuerpos y sexualidad, impactando negativamente en la calidad de vida de las mujeres.

Posteriormente a esta ley, países como Argentina en el 2009 y Panamá en el 2013 han incluido la "violencia obstétrica" en sus legislaciones en torno a la protección integral a las mujeres; Bolivia la integra bajo el concepto de "violencia en los servicios de salud", en tanto que otros países como Uruguay y Colombia han incluido respectivamente normas y regulaciones administrativas en torno al "parto humanizado" (2).

La Organización Mundial de la Salud (OMS) (3) produjo en el 2014 la declaración para la "Prevención y erradicación de la falta de respeto y el 
maltrato durante la atención del parto en centros de salud", en la que enfatiza en la necesidad de desarrollar investigación orientada a "definir y medir el maltrato, y la falta de respeto, en los centros de salud públicos y privados de todo el mundo, y para comprender mejor su impacto en las experiencias y las elecciones de salud de las mujeres".

El propósito del presente trabajo ha sido elaborar, a partir de una búsqueda en bases de datos, un estado del arte que presenta los avances de la investigación reciente de las ciencias sociales en América Latina sobre violencia obstétrica, con el interés de hacer un aporte a su problematización. Las categorías generales consideradas para abordar el análisis fueron las siguientes: (a) contextos en que se produce la investigación en ciencias sociales sobre violencia obstétrica; (b) construcción conceptual sobre la violencia obstétrica; (c) modelos explicativos de los orígenes de la violencia obstétrica y; (d) alternativas para un cambio de la situación. A partir del recorrido realizado, se desarrolla un aparte sobre la interpretación que hacen los autores de este trabajo acerca de los hallazgos obtenidos y se destaca la proyección futura que se devela para la investigación y la acción en este campo.

\section{Descripción del proceso metodológico}

Un estado del arte es un proceso sistemático y organizado de tipo hermenéutico que da cuenta de los avances de la investigación en determinado ámbito conceptual, en cuya construcción se ve implicado un ejercicio de contextualización, ordenamiento e interpretación de la información. Este tipo de investigación está constituida por las siguientes etapas: definición de descriptores, criterios de búsqueda, recolección, depuración y clasificación de la información y análisis e interpretación de los datos. En el estado del arte se pueden identificar dos procesos generales, según Londoño et al. (4):

a). La búsqueda, selección, organización y disposición de fuentes de información.

b). La integración de la información que conlleva el análisis de los textos contenidos en las fuentes, que corresponde a la dimensión hermenéutica del proceso.

El proceso de indagación desarrollado para la construcción del estado del arte fue realizado entre julio del 2017 y enero del 2018 y estuvo construido partiendo de una búsqueda de la literatura científica en español e inglés, producida en países de América Latina entre el 2010 y el 2017, teniendo 
en cuenta las bases de datos Clacso, Dialnet, Redalyc, Scielo, Ciencias sociales y Humanas (CUC), Humanidades y Ciencias sociales (SIC), Clase, Base de datos bibliográfica de América Latina (Birla) y Unesco, además de repositorios institucionales. Como criterios de inclusión se establecieron los siguientes: documentos de investigación teórica y aplicada en ciencias sociales, escritos en inglés y español, que reporten estudios publicados en América Latina entre el 2010 y el 2017, que aborden la violencia obstétrica de manera explícita o implícita. Los criterios para la exclusión de los documentos fueron definidos así: estudios no estructurados científicamente o que no aporten datos de interés para la investigación.

Los siguientes descriptores orientaron la búsqueda general: violencia obstétrica, violencia ginecológica, parto violentado, ciencias sociales, América Latina. La búsqueda dio como resultado 5440 documentos de los cuales fueron revisados 34 que cumplían con los criterios de inclusión. Finalmente se obtuvieron 18, al aplicar los criterios de exclusión (14 artículos, tres ponencias y un trabajo de posgrado).

Se implementó un análisis documental, utilizando la técnica de fichaje propuesta por Montero y Hachman (5). Una vez obtenida la información, se realizó un proceso hermenéutico de análisis y codificación, partiendo de las categorías generales establecidas, seguido por la elaboración de cuadros analíticos y mapas conceptuales que permitieron el ordenamiento de los hallazgos para proceder a su interpretación y a la posterior construcción del documento.

\section{Sobre los estudios revisados y los contextos de donde provienen}

De los dieciocho documentos revisados, ocho se centran en la problematización de la "violencia obstétrica" (también "violencia ginecológica") y diez presentan aproximaciones que sirven a la comprensión del fenómeno o a la profundización de sus dimensiones, hallándose, entre otros, títulos referidos al control del nacimiento, al habitus médico autoritario y al parto medicalizado.

Trece de los trabajos analizados son de tipo teórico y cinco de investigación de corte cualitativo, en que se hizo uso de técnicas como la etnografía (incluida la autoetnografía), los grupos focales y las entrevistas focalizadas o semiestructuradas. Priman las aproximaciones de corte crítico-reflexivo y los análisis de situación con perspectiva de género y derechos; también se orienta 
la reflexión desde una visión crítica enfocada en la asimetría del saber-poder presente en la relación entre profesionales de la salud y gestantes.

Las investigaciones provienen sobre todo de la antropología y la sociología, además de que se encuentran aportes desde la psicología social, la educación y el trabajo social. Entre los países de la región que mayores contribuciones hacen al estudio de la violencia obstétrica desde las ciencias sociales se encuentran México y Argentina. Otros países de donde surgen estudios en este campo son Venezuela, Costa Rica, Chile, Brasil, Uruguay y Colombia.

En lo referente a los ámbitos de donde proviene la investigación sobre violencia obstétrica, la academia resulta ser el contexto donde por excelencia se produjeron los aportes analizados, cuando de los dieciocho documentos, diecisiete fueron elaborados por miembros de grupos de investigación, docentes y estudiantes de maestría y doctorado de universidades de la región.

\section{La violencia obstétrica como concepto}

De la conceptualización sobre la violencia obstétrica se devela la existencia de un fenómeno multideterminado que conlleva la afectación de los derechos humanos de las mujeres vía una atención en salud sexual y reproductiva autoritaria y centrada en relaciones asimétricas de poder, dentro de un contexto altamente jerarquizado que origina la pérdida de autonomía sobre sus cuerpos y en que ellas son convocadas a someterse al poder institucional y pierden el control de un proceso que les pertenece.

\section{Violencia de género y violencia institucional}

La violencia obstétrica se concibe como una forma específica de violencia de género contra las mujeres (6-8) que implica un trato deshumanizado y la medicalización y la patologización de los procesos reproductivos (9-11) y conlleva la apropiación de los cuerpos de las mujeres $(8,12,13)$. En este tipo de violencia se presenta un control de la capacidad reproductiva y la sexualidad de las mujeres $(6,8,10,13)$, y son los miembros del personal de salud quienes la ejercen (7-9,13-15).

La mayoría de los autores se refieren a que la violencia obstétrica se da durante el trabajo de parto y el parto; no obstante, algunas autoras consideran que se puede presentar a lo largo del embarazo, parto y puerperio $(10,16,17)$. 
La afectación de los derechos humanos (14) y de los derechos sexuales y reproductivos $(7,13,14)$ es reportada en buen número de las aproximaciones. Por último, las y los autores mencionan que la violencia obstétrica reduce la capacidad de decisión de las mujeres $(6-8,13)$ y su calidad de vida $(8,13)$.

Algunos autores y autoras definen la violencia obstétrica como un fenómeno multifactorial que surge de la intersección entre la violencia estructural de género y la violencia institucional en salud $(7,8)$. De igual modo, se afirma que esta forma de violencia se manifiesta en prácticas cotidianas $(6,11)$ que se ejercen en servicios de salud tanto públicos como privados (6,7). Entre los derechos humanos y reproductivos que se ven afectados con la violencia de tipo obstétrico, se destacan, de acuerdo con lo que establece Almonte (6), la igualdad, la no discriminación, la información, la integridad, la salud y la autonomía reproductiva.

\section{Mecanismos de control y opresión}

Para quienes son más afines a la perspectiva foucaultiana, la violencia obstétrica es un producto ideológico, basado en una hegemonía del saber/poder que detentan los profesionales médicos y en una apropiación del cuerpo de las mujeres a través de un sistema de salud altamente jerarquizado $(9,10)$. Según lo expuesto por Arguedas (10), la violencia obstétrica se puede considerar un mecanismo de control y opresión que proviene del ejercicio del "poder obstétrico", el cual constituye una forma de poder disciplinario que, siguiendo a Foucault, produce cuerpos femeninos "dóciles" que sirven a la potencialización de la efectividad de embarazos, partos y cuidado de los recién nacidos.

La violencia obstétrica, tal cual la conciben buena parte de los investigadores cuyos trabajos han sido analizados, está configurada a partir del ejercicio de mecanismos de control $(9,10,13)$ y de opresión (12), en un contexto donde se presentan unas relaciones de poder asimétricas entre el personal de salud y la gestante $(6,7)$ y una atención marcada por el autoritarismo (7).

Se instaura, de acuerdo con Castrillo (17), como una forma de relación de dominación /subordinación de las y los pacientes por la figura del médico como portador del saber-poder. Las relaciones de poder asimétricas y las imposiciones de clase y jerarquías presentes en la violencia obstétrica suponen, entre otras cosas, a las pacientes como sujetos subalternados, presentándose 
condiciones para que se desplieguen violencias, no solo sobre las mujeres, sino sobre los varones que son sus parejas y sobre los recién nacidos.

La atención médica del parto se asienta sobre un control simbólico del saber en el que se desconoce la información y el conocimiento que poseen las mujeres sobre su proceso de parto, lo cual se estructura como una forma de poder simbólico que invalida sus percepciones y decisiones y anula sus saberes $(6,17)$. Se promueve la conformidad y la docilidad de las mujeres, quienes pierden su protagonismo en el parto para resultar siendo, según lo señala Sadler (8), quienes "colaboran con el personal médico y le ayudan a realizar su trabajo". La tolerancia al dolor durante el trabajo de parto se convierte en exigencia moral a la mujer para asumir su papel de madre con calidad y aptitud, como lo menciona Arguedas (10).

\section{Homogenización de las gestantes}

La homogenización de las gestantes, que las convierte en un número más (8), y su confinamiento al papel de espectadoras de lo que ocurre en su propio cuerpo $(10,13)$ son situaciones asociadas a la deshumanización que caracteriza la violencia obstétrica. El parto se asume como un evento externo a ellas que se protocoliza/estandariza por igual para todas las gestantes, sin reconocer individualidades (18).

\section{Violencias invisibles}

Con respecto a su tipificación, Almonte (6) señala que la violencia obstétrica se presenta en dos tipologías: (a) física: manipulación del cuerpo, prácticas invasivas, suministro de medicamentos no justificados y modificación de los procesos biológicos de la mujer sin que ella otorgue su consentimiento y, (b) psicológica: "regaños, burlas, ironías, insultos, amenazas, humillaciones, manipulación de la información, negación al tratamiento [...] utilizarlas como recurso didáctico sin ningún respeto a su dignidad humana".

Varias de las prácticas que se realizan y que afectan los derechos de las mujeres durante el parto han sido consideradas innecesarias por la evidencia científica. De acuerdo con el trabajo etnográfico realizado por Sadler (8) en Chile, estas son prácticas que se han institucionalizado: 
Se inmoviliza a las mujeres en camas con monitores fetales y vías venosas, se les impide la movilidad durante el trabajo de parto, se ponen trabas al acompañamiento continuo de personas externas al personal de salud, la mayoría de los nacimientos ocurre en posición litotómica, y los bebés se separan de sus madres para exámenes y chequeos de rutina.

García-Jordá y sus colegas (19), a partir de su estudio cualitativo en un hospital de La Habana, hallaron que se ejercen violencias físicas y de género a lo largo de la atención reproductiva. Durante el trabajo de parto, las autoras observaron lo siguiente:

Las mujeres permanecieron con las batas abiertas, descubiertas ante la presencia de personal médico y no médico [...] La exploración y observación médica fue constante ante la inminencia del parto y por lo general por varios profesionales, obstetras, residentes, estudiantes, enfermeros que rodearon a las mujeres. "No puedo más" fue la expresión más frecuente por parte de ellas.

Vallana, con base en su aproximación cualitativa con mujeres que hablaron sobre sus embarazos y partos en diferentes contextos institucionales de Bogotá y partiendo de sus propias vivencias, hace énfasis en la permanente apropiación del cuerpo de las gestantes que caracteriza a la experiencia del parto hospitalario y en la poca sensibilidad por parte del personal de salud frente a lo que ellas puedan sentir:

Tocar, retocar, mirar, observar, examinar, hurgar, oler [...] todo está -permitido y naturalizado como "adecuado" en el contexto del parto hospitalario, percibiéndose un profundo desinterés por aspectos emocionales y psicosociales como el pudor de las parturientas.

La violencia obstétrica se halla naturalizada y por lo tanto es invisibilizada con frecuencia en los países de América Latina. Es poco reconocida y poco reportada, según lo menciona Almonte (6). De acuerdo con Müller y sus colegas (19), cuestionar el nacimiento resulta ser un tema tabú que genera resistencias y tensiones, incluso en ámbitos del conocimiento.

Belli (9) señala que a menudo las formas de proceder en las instituciones de salud que se pueden considerar violentas son vistas como "normales" por las mismas mujeres, sobre todo por aquellas que son atendidas en servicios no 
pagos quienes asumen que ser sometidas a tratos poco amables hace parte de hacer uso de la atención gratuita. Según García-Jordá y sus colegas (18), las mujeres cubanas entrevistadas en su estudio se autocensuraron cuando describieron momentos de vulnerabilidad durante sus partos que las llevaron a alterarse o a pedir una cesárea; no mostraron comprensión de la razón por la cual se realizaron varios de los procedimientos, pero los vieron necesarios y los consideraron favorables para el bienestar de sus bebés; en el proceso se les instó a "portarse bien”, a ser "disciplinadas" y a "no gritar”. Müller y sus colegas (20) añaden que a pesar de que pueda presentarse malestar en ellas, si las mujeres no conocen otras posibilidades de atención del parto será difícil que se adhieran a un discurso de reivindicación de un nuevo modelo obstétrico.

\section{Otras víctimas de la violencia obstétrica}

Además de las mujeres, de sus parejas y los recién nacidos, otras víctimas de la violencia obstétrica, que descubre Magnone (12), son las personas que hacen parte del equipo de salud que buscan poner en práctica el modelo de humanización, quienes resultan atacadas por el sistema. De acuerdo con lo señalado por Campiglia (11), médicos y enfermeras que hacen parte del dispositivo ginecobstétrico y se desmarcan de este suelen ser castigados. Magnone sugiere generar cambios en las estructuras de poder institucionales que están desdibujando el acto asistencial (21).

\section{Modelos explicativos de la violencia obstétrica}

Para explicar la violencia obstétrica, los autores toman básicamente dos caminos: el primero, se instaura en los mundos intersubjetivos donde desempeñan un papel central las relaciones asimétricas de poder y de género que se dan entre el personal médico y la paciente; y el segundo, encuentra el desequilibrio en el modelo tecnocrático que, al darle mayor peso a la productividad, afecta el sistema de salud.

\section{Violencia obstétrica: una cuestión de relaciones de poder}

En la violencia obstétrica se destaca como uno de sus puntos de partida más relevantes el paradigma médico jerarquizado donde se presentan unas relaciones asimétricas entre los profesionales de la salud y las pacientes $(6,9,15)$. Belli afirma (9) que la violencia obstétrica constituye un resultado prácticamente inevitable de la aplicación del paradigma médico vigente en buena parte de los países de América Latina. 
Los orígenes de este modo de violencia se explican por el patriarcado, que se hace presente en el ámbito médico, deriva en androcentrismo y sexismo y lleva a una asimetría en cuanto a las relaciones saber-poder que favorece la implementación del paradigma tecnocrático de atención, centrado en el control sobre el potencial reproductivo de las mujeres. Arguedas (10) afirma que en la violencia obstétrica es posible detectar una forma específica de poder disciplinario, vinculada con la estructura social de género característica del sistema patriarcal, y añade que "este poder se ejerce en un territorio de género específico: los cuerpos que pueden embarazarse y parir”.

Según Castro (7), las relaciones de poder que se ponen en juego en el mundo médico se construyen a partir de un "habitus médico" formado desde la educación en las facultades de medicina a través de unas relaciones jerarquizadas y autoritarias, que se reproduce en las formas de interacción dentro del equipo médico y en las relaciones entre los miembros del personal de salud y las mujeres en que el distanciamiento con las pacientes se da “como una forma de protección de sí mismos/as". Castrillo (17) señala que la práctica médica es un escenario que, por sus interacciones como práctica social, favorece que se presente la violencia obstétrica:

La práctica médica en tanto práctica social (y no técnico-inocua) expresa relaciones de poder, posiciones de clase, jerarquías, incluso sujeción y formas de violencia, y supone, entre otras cosas, a los pacientes como sujetos subalternizados. En esas relaciones de poder asimétricas se dan condiciones para que se ejerzan violencias sobre las mujeres, los varones y los recién nacidos.

\section{Violencia obstétrica como derivación del modelo tecnocrático del nacimiento}

Otra realidad que los autores conectan con la violencia obstétrica es el hecho de que la atención en salud se ve afectada por las carencias del sistema sanitario y la centralidad de este en la productividad. Según lo expuesto por Vallana, la prioridad está en atender el mayor número de partos, sin que importe su calidad, asemejándose la atención a una cadena productiva nominada por Davis-Floyd (16), en 1993, como "modelo tecnocrático del nacimiento". Este modelo crea una ruptura con el conocimiento ancestral, que viene siendo transmitido y heredado de una a otra generación lo que, como lo expone Sadler, lleva a una polarización entre el parto intervenido 
y los modelos asociados al parto natural, creando la desvalorización de los segundos y anulando el diálogo de saberes (8).

\section{Alternativas para el cambio de situación}

Entre las alternativas que surgen a partir de los avances en la investigación social latinoamericana considerados en este proceso de abordaje a la violencia obstétrica, se destaca la importancia de trabajar con el personal médico, bien sea gestando cambios estructurales en su educación para generar un habitus menos autoritario y fortaleciendo su formación ética (7) o, promoviendo una atención humanizada a partir de sus propias necesidades y circunstancias $(9,22)$. De igual modo, se propone ahondar en sus perspectivas y realidades cotidianas, como modo de comprender el fenómeno a profundidad, superando los juicios normativos basados en información superficial.

\section{Reflexión y sensibilización entre los miembros de equipo de salud}

Belli (9) sugiere repensar el papel de los miembros del personal de salud como victimarios, sin considerar que son a la vez víctimas del sistema. Así, expone que en tanto se les observe como meros perpetradores del acto violento, y no como piezas de un mecanismo complejo que los trasciende, la reflexión en torno al tema resultará inútil. Es perentorio suscitar reflexión y sensibilización en los agentes de salud para poder cambiar aquellas prácticas que violan los derechos de las mujeres, pero se requiere desarrollar sensibilidad ante sus necesidades si se desea contar con médicos y enfermeras dispuestos a cambiar. En la misma línea, Pozzio (22) propone profundizar en las prácticas concretas de los médicos y médicas y sus formas de negociar, comprender y reaccionar en su actuar cotidiano, buscando abordar el modo como se generan sus relaciones y estructuras, para lograr una comprensión profunda del fenómeno, sin juicios a priori y sin condenas basadas en una mirada superficial de la problemática.

\section{Revisión de las condiciones laborales del personal médico}

Vallana (16) se refiere a la importancia de revisar las condiciones laborales de los profesionales de salud y reconocer sus circunstancias:

Es necesario analizar las condiciones, los horarios de trabajo y las recargas laborales de las profesionales de la salud, cuyo trabajo requiere de 
paciencia, descanso, tranquilidad y una muy alta responsabilidad. Un/a profesional extenuado/a, con una sala de partos llena y el acoso de la patología en mente, responde sin paciencia ante el llanto y los gritos de dolor, es un profesional asustado ante la eventualidad de la patología y la amenaza de la demanda, e inseguro ante sus procedimientos y actuaciones. En estas circunstancias, el personal de la salud tiene tanto miedo como las parturientas, pero también un poder que puede traducirse en una experiencia obstétrica en el mejor día de la vida de otro ser humano.

\section{Nuevos modelos asistenciales}

Otras recomendaciones se centran en el cambio de paradigmas en la atención del parto. Así, García-Jordá y sus colegas (18) consideran imperativo buscar opciones fundamentadas en modelos asistenciales no hegemónicos para la atención al parto, en que se consideren las preferencias individuales. Sadler (8), por su parte, propone devolver a las mujeres su lugar en los procesos reproductivos y respetar en ellas, sus recién nacidos y familias, las dimensiones psicológicas, afectivas, sociales y culturales de sus experiencias.

\section{Construcción de ciudadanía reproductiva}

Castro (7) considera esencial una transformación de las relaciones médico-paciente, propendiendo por la construcción de ciudadanía en ese intercambio. En lo relativo a la investigación, Castro y Erviti (14) exponen que la conceptualización que se hace de la violencia obstétrica en el campo médico como un problema de "calidad de la atención" constituye un obstáculo para su abordaje, siendo necesario que se construya como objeto de estudio desde un enfoque de género y derechos, con el apoyo de conceptos sociológicos como el de habitus profesional y el de campo médico, ya que la violación de derechos reproductivos de las mujeres es un epifenómeno del orden social y de género dominante y de la estructura de poder propia del campo médico. Proponen incluir el concepto de ciudadanía reproductiva como "la capacidad de las mujeres de apropiarse, ejercer y defender sus derechos en materia de salud sexual y reproductiva".

Gainza y sus colegas (23) avanzan hacia "la autonomía de las mujeres como seres sexuales y como ciudadanas". En este sentido, se requiere una apropiación por parte de ellas de su capacidad reproductiva, garantizando condiciones que les permitan escogerla y vivenciarla libremente. Las autoras insisten en la importancia de asumir el cuerpo como concepto político y de 
reconceptualizar la "ciudadanía en el cuerpo" como categoría política por cuanto las definiciones generalizadas de ciudadanía suelen no contemplar la dimensión subjetiva.

\section{Sobre los hallazgos: algunas reflexiones}

Son tres las tendencias conceptuales para el abordaje a la violencia obstétrica que se visualizan a lo largo de este recorrido: (a) el enfoque de género y derechos, desde donde se destaca la violación de los derechos sexuales y reproductivos y otras formas de afectación de los derechos de las mujeres y de su construcción de ciudadanía, además de que se considera la violencia obstétrica como una expresión de la violencia de género de tipo institucional y de injusticia contra las mujeres; (b) la crítica feminista de corte foucaultiano, que asume la violencia obstétrica como instrumento de poder, ligado a la naturalización y medicalización de los procesos reproductivos de las mujeres y a la biopolítica, generadora de control y apropiación del cuerpo de las mujeres en la práctica de la salud sexual y reproductiva, la cual se ve estrechamente ligada al orden sexista y patriarcal; y (c) la mirada bioética, que enfatiza en la deshumanización de las tecnologías en reproducción y que refleja insuficiencias de los sistemas de salud, centrados en la productividad, además de reconocer una construcción cultural del quehacer médico y de las relaciones en el ámbito institucional que ha venido perdiendo su norte.

La violencia obstétrica se recrudece a partir de la tecnificación de los procesos reproductivos, con la cual los sistemas de salud han buscado, entre otros efectos, minimizar la mortalidad materna. Los hallazgos de este estudio llevan a plantear que en el ámbito de la atención en salud reproductiva se presenta una tensión entre la visión de las mujeres como objeto de cuidado y la visión de las mujeres como sujetos de derecho, la cual requiere ser enfrentada ayudando a las mujeres a rescatar su lugar como protagonistas de sus embarazos, partos y postpartos, sin dejar de lado los máximos niveles del cuidado requeridos. Para ello es necesario desarrollar cambios estructurales en el sistema, trabajar por la transformación de la cultura médica e instaurar políticas de salud orientadas a mejorar las condiciones de todos los actores y actoras implicados en la salud sexual y reproductiva.

La investigación social requiere necesariamente volcarse hacia el reconocimiento de la perspectiva de las mujeres y del personal de salud, cuyas experiencias y necesidades deben hacer parte central del análisis sobre 
la violencia obstétrica y sus posibles soluciones en los países de América Latina. La vulnerabilidad social de las mujeres ha mostrado ser un aspecto estrechamente ligado a la experiencia de la violencia de género de tipo institucional. Si bien algunos estudios analizados en este recorrido la reconocen, es de importancia vital profundizar en ella en futuros estudios sobre violencia obstétrica. Siendo la realidad del aborto un aspecto central del debate en torno a los derechos sexuales y reproductivos de las mujeres en América Latina, que suscita tensiones y violencias de género de carácter institucional, se observa un vacío de la investigación en torno a esta realidad en los estudios revisados, el cual requiere ser resuelto.

\section{A manera de conclusión}

La violencia obstétrica como objeto de estudio en el campo de las ciencias sociales en América Latina ofrece un panorama de inmensa riqueza e interés, que invita a una reflexión profunda sobre la deshumanización del modelo tecnocrático de la atención en salud reproductiva, cuyas implicaciones negativas para las mujeres y sus derechos son inocultables, a la luz de los avances expuestos.

Es necesario un cambio de paradigmas en los sistemas de salud, en los procedimientos y en la formación de las y los profesionales con miras a la humanización de la atención en el campo de la salud de las mujeres y la erradicación de todas las formas de la violencia obstétrica, para que sea posible hablar de ejercicio de ciudadanía en ese ámbito. Para ello, es preciso trabajar en un proceso de doble vía que, de una parte, involucre los aspectos intersubjetivos inmersos en la práctica cotidiana de la salud sexual y reproductiva y, de otra, las estructuras institucionales que requieren ser reordenadas y trasformadas.

La investigación social está llamada a avanzar en el levantamiento del escenario donde ocurre la violencia obstétrica, profundizando en las vivencias de las mujeres que hacen parte de los grupos sociales mayormente vulnerables y explorando la mirada de los miembros del personal de salud y los contextos en que estos se desenvuelven. De igual modo, es importante reconocer el aborto como un terreno de indagación de importancia crucial en este campo. 
La inclusión de una mirada de género y de derechos y el cuestionamiento profundo, desde sus orígenes, de las relaciones asimétricas de poder inmersas en los hechos de la salud reproductiva, aunados a cambios sustanciales en los modelos de salud, constituyen un imperativo ético en el trayecto por seguir para el rescate del protagonismo de las mujeres en sus embarazos, partos y maternidades, en un diálogo permanente de los diferentes actores de los servicios de salud con sus saberes, necesidades y decisiones.

\section{Referencias}

1. Fondo de Población de las Naciones Unidas (UNFPA). Ley Orgánica sobre el derecho de las mujeres a una vida sin violencia [Internet] 2006. [citado 2017 jul. 6]. Disponible en: http://venezuela.unfpa.org/doumentos/Ley_mujer.pdf

2. Gherardi N. Otras formas de violencia a las mujeres qué reconocer, nombrar y visibilizar. Santiago: Naciones Unidas-CEPAL; 2016.

3. Organización Mundial de la Salud. Prevención y erradicación de la falta de respeto y el maltrato durante la atención del parto en centros de salud. Declaración de la OMS. Ginebra: OMS; 2014.

4. Londoño OL, Maldonado LF, Villafañez LC. Guía para construir estados del arte. International Corporation of Networks of Knowledge [Internet]. 2014 [citado 2017 nov. 9]. Disponible en: http://www.colombiaaprende.edu.co/html/investigadores/1609/articles-322806_recurso_1.pdf

5. Montero M. Hochman E. Investigación documental, técnicas y procedimientos. Caracas: Editorial Panapo; 2005.

6. Almonte, G. Violencia ginecológica y obstétrica. Revista Décsir. EAM [Internet]. 2016 [citado 2018 feb. 3]2:1-10. Disponible en: http://decsir.com.mx/wp-content/ uploads/2016/03/1-indagaciones.pdf

7. Castro R. Génesis y práctica del habitus médico autoritario en México. Revista Mexicana de Sociología. 2014;76(2):167-97. https://www.redalyc.org/articulo. oa? $\mathrm{id}=32130485002$

8. Sadler M. Etnografías del control del nacimiento en el Chile contemporáneo. Revista Chilena de Antropología [Internet]. 2016 [citado 2017 jul. 5];33:45-57. Disponible en: https://www.researchgate.net/publication/309034638_Etnografias_ del_Control_del_Nacimiento_en_el_Chile_Contemporaneo.

9. Belli LF. La violencia obstétrica: otra forma de violación a los derechos humanos. Revista Redbioética/Unesco. 2013;4(7):25-34. Disponible en: https://ri.conicet. gov.ar/handle/11336/12868 
10. Arguedas G. La violencia obstétrica: propuesta conceptual a partir de la experiencia costarricense. Cuadernos Intercambio sobre Centroamérica y el Caribe [Internet]. 2014 [citado 2017 nov. 29];11(1):145-69. Disponible en: https://revistas. ucr.ac.cr/index.php/intercambio/article/view/14238

11. Campiglia M. Violentar el nacimiento. El Cotidiano [Internet]. 2015 [citado 2017 nov. 27];(191):79-86. Disponible en: http://www.elcotidianoenlinea.com. $\mathrm{mx} / \mathrm{pdf} / 19109$.pdf.

12. Magnone Alemán N. Derechos sexuales y reproductivos en tensión: intervencionismo y violencia obstétrica. Trabajo presentado en las X Jornadas de Investigación de la Facultad de Ciencias Sociales [Internet]. Montevideo: UdelaR; 2011 [citado 2014 jun. 28] Disponible en: http://www.fcs.edu.uy/archivos/Magnone.pdf

13. Camacaro M. La violencia obstétrica como cuestión de los derechos sexuales y reproductivos de las mujeres. Revista Venezolana de Estudios de la Mujer. 2013;18(40):185-92. Disponible en: http://saber.ucv.ve/ojs/index.php/rev_vem/ article/view/5343

14. Castro R, Erviti J. 25 años de investigación sobre violencia obstétrica en México. Revista Conamed [Internet]. 2014 [citado 2017 jul. 9];19(1):37-42. Disponible en: http://biblat.unam.mx/es/revista/revista-conamed/articulo/25-anos-de-investigacion-sobre-violencia-obstetrica-en-mexico.

15. Castrillo AB. La perspectiva de género en el estudio de la intervención médica en el parto. Memoria Académica PostCongreso de Investigación Cualitativa en Ciencias Sociales, Córdoba, Argentina [Internet] 2014 oct. 2-3 [citado 2017 jul. 4]. Disponible en: http://www.memoria.fahce.unlp.edu.ar/trab_eventos/ev.4125/ ev.4125.pdf

16. Vallana, V. Parirás con dolor, lo embarazoso de la práctica obstétrica. Discursos y prácticas que naturalizan la violencia obstétrica en Bogotá [trabajo de grado de maestría en Estudios Culturales]. Bogotá: Pontificia Universidad Javeriana; 2016.

17. Castrillo MB. Dime quién lo define y te diré si es violento. Sex, Salud Soc (Rio J.) [Internet]. 2016 [citado 2017 jun. 20 ];24:43-68. Disponible en: http://www. scielo.br/pdf/sess/n24/1984-6487-sess-24-00043.pdf.

18. García Jordá D, Díaz Bernal Z, Acosta Álamo M. Perspectiva antropológica y de género en el análisis de la atención al embarazo, parto y puerperio. Revista Cubana Salud Pública [Internet]. 2010 [citado 2016 oct. 16];36(4). Disponible en: http://www.redalyc.org/articulo.oa?id=21416138007

19. García Jordá D, Díaz Bernal Z, Acosta Álamo M. El nacimiento en Cuba: análisis de la experiencia del parto medicalizado desde una perspectiva antropológica. Ciencia \& Saude Colectiva. 2012;17(7):1893-902. Disponible en: http://scielo.sld. cu/scielo.php?script =sci_arttext\&pid $=$ S0864-34662013000400009 
20. Müller E, Rodrigues L, Pimentel C. O tabu do parto. Dilemas e interdições de um campo ainda em construção. Civitas. 2015;15(2): 272-93. doi: 10.15448/19847289.2015.2.17928.

21. Magnone-Alemán N. Entre lo formal y lo sustantivo: La calidad de la asistencia al parto en el Uruguay. Sexualidad, Salud y Sociedad [Internet]. 2017 [citado 2018 jul. 30] (27):97-117. Disponible en: http://www.e-publicacoes.uerj.br/index. php/SexualidadSaludySociedad/article/view/28707/22726

22. Pozzio MR. La gineco-obstetricia en México: entre el "parto humanizado" y la violencia obstétrica. Estudos Feministas, Florianópolis [Internet]. 2016 [citado 2018 feb. 15];24(1):101-17. Disponible en: http://www.scielo.br/scielo.php?pi$\mathrm{d}=$ S0104-026X2016000100101\&script $=$ sci_abstract\&tlng $=$ es

23. Gainza P, Labastie M, Magnone-Alemán N. La atención contemporánea del parto: jaque a la perspectiva de derechos humanos. Uluá Revista de Historia, Sociedad y Cultura [Internet]. 2013 [citado 2018 feb. 15];(22): 175-96. Disponible en: http:// revistas.uv.mx/index.php/ulua/article/view/1187 